\title{
Pharmacogenomics: The Significance of Genetics in the Metabolism of Natural Medicines
}

\author{
Nancy W. Hanna \\ College of Pharmacy, California Northstate University, Rancho Cordova, USA. \\ Email: nancywhanna@gmail.com
}

Received June $13^{\text {th }}, 2012$; revised July $23^{\text {rd }}, 2012$; accepted August $15^{\text {th }}, 2012$

\begin{abstract}
Natural products have been implemented in medicine through use as herbal medications, chemical compound extraction for prescription medication, or a natural source of food to fight various infections and diseases. Genetics has played a role in identifying various interactions between existing drugs and side effects. In addition, various food allergies have been identified with children in recent years, suggesting genetic associations between certain populations carrying specific genetic alleles. The recent availability of genomic data and our increased understanding of the effects of genetic variations permit a quantitative examination of the contribution of genetic variation to efficacy or toxicity of compounds derived from natural sources. The identification of target molecules relevant for diseases allows screening for natural products capable of inhibiting targets which can lead to the development of rational treatment of various diseases including neurobiological disorders, cancer, osteoporosis, and cardiovascular diseases. This allows for more opportunities to predict the response of individual patients. Identification of genetic variations that arose as a consequence of naturally occurring compounds will help identify gene alleles, or protein ligands that can affect the pharmacodynamic and pharmacokinetics of the natural products in question. In addition, diet modification and precautions to food products can be identified to help consumers limit or increase certain food intake. Understanding the molecular mechanisms underlying these interactions and their modification by genetic variation is expected to result in the development of new drugs that optimize individual health. We expect that strategies for individualized therapies will lead to improved results for patients.
\end{abstract}

Keywords: Pharmacogenomics; Natural Medicine; Polyphenols; Lycopenes; Folic Acid; Tobacco; Vitamin E; Curcumin; Soy; Vitamin D; Digoxin

\section{Introduction}

Genomics is now playing a major role in identifying information from the human body and applying it to current drug therapy. It has produced a new era of individualized drug therapy for patients to achieve higher efficacy and safety. Due to the enhanced development of today's technologies, the long-term benefits of pharmacotherapy management are becoming closer to implement in clinical practice [1].

It has been recognized through several healthcare management facilities that individuals respond differently to medications, and the correlation of the drug medication to the efficacy has led more pressure on physicians and pharmacists to apply the concept of personalized medicine into clinical practice [1]. However, this new direction is only possible with the support of pharmaceutical industry research to conduct clinical trials on the pharmacotyping of medications, which can also help researchers cut on the expenses of medications through conducting more tailored and smaller clinical trials [1,2]. Tailored clinical trials have also proposed several issues with misinterpretation of genetic factors, which shows that highly specific genetic tests are needed to eliminate any false positives or false negatives in the laboratory setting [2].

The concept of pharmacotherapy has been influenced through previous research of associating populations with respect to their geographical, ethnic, or racial background in regards to their disease disposition or intelligence. In return, pharmacotherapy research has taken this association to correlate genetic response to medications $[1,2]$.

Natural product use has been valued for thousands of years through cave site engraftments or historical documentation. The search for new medicinal products has been extrapolated from plants, animals, and micro-organisms, with historical evidence suggesting the use and benefits of natural products [3]. It has led the pharmaceu- 
tical industry to several plant-derived medications through modern analytical and structural analysis of plant-based compounds $[3,4]$. The discovery of morphine in 1805 by German pharmacist Fredrich Wilhelm Sertürner (17831841) became the first plant-based medical discovery and influenced several natural-based medical discoveries [3].

Natural products have offered several advantages to their use in drug manufacture due to the ease of synthesizing chemical compounds which lowered the cost of drug production [3]. In addition, their use in society has been easily targeted through its habitual interaction in nature, such as bacterial suppression, or natural defense against herbivores, which led drug discoveries in antimicrobials, laxatives, and muscle relaxants [3]. Comparative studies between animals and plants has revealed that both species use similar signaling molecules and recaptors, suggesting that plant chemical compounds have the ability to bind to human disease to modulate binding and metabolism [3]. Nearly $50 \%$ of the drugs introduced in the past 20 years have been derived directly or indirectly from natural products, and several in vitro tests such as medium pressure solid-liquid extraction (MPSLE), rotation planar extraction (RPE), and thin layer chromatography (TLC) are currently available for screening plants and their properties for drug extrapolation and investigation [4]. Despite this fact, very little effort has been made to looking at the influence of pharmacogenetics in the efficacy and toxicity of natural medicines.

Current research of the pharmacogenomics of natural products is limited, yet provides a great advancement and potential for the standard use of genetic screening during pharmaceutical production of natural products. For natural products that are in the early stages of drug development, more research should be conducted to determine if gene variants are responsible for the differences in effects and toxicity profiles between patients. If genetic variants are responsible for the different responses, pharmacogenomic tests may be used to determine who will actually benefit from the drug. In addition, polymorphisms in the genes for drug-metabolizing enzymes and transporters may influence the efficacy of natural medicines mediated through these pathways.

\section{Cancer Therapy}

\subsection{Tea Polyphenols}

Obesity has been regarded as one of the major risk factors and threats for several disease states including cardiovascular diseases and cancers. It can lead to the overactivation of fatty enzymes in the body leading to slow metabolism and blood clot formation in cardiovascular health, or enzyme activation in cancer [5]. Fatty acid synthase (FAS) is an important enzyme in human metabolism, and studies have shown that the expression of
FAS is highly increased in human cancer cells, including breast, colon, ovary, lung and prostate cancer. The protein hormone, leptin, plays a major role in regulating FAS by inhibiting its function in the human body; however, in some patients, FAS has evolved showing resistant strains to leptin [5]. It has led to the research and development of leptin-like compounds, known as FASinhibitors, which produce cytotoxic effects similar to leptin by increasing malonyl-CoA levels in the hypothalamic neuron [5].

Several investigators have looked for natural trends in obesity and populations, and one has led to the discovery of lower obesity rates in vegetarian populations such as Asian populations, where black and green tea-drinking in highly popular.

Tea polyphenols have shown previous clinical benefit through antioxidant properties and scavenging effects of radical oxygen species, which lead to several diseases including cancers. In order to understand the genetic background of tea polyphenols and possible contribution to anticancer properties, chemical compounds have been extrapolated from both green and black tea to understand the possible chemical contribution to lower obesity rates. According to C.-W. Yeh et al., green tea contains six catechins, $(+)$ catechin $(C),(-)$-epicatechin $(\mathrm{EC}),(-)$-epicatechin 3-gallate (ECG), (-)-epigallocatechin (EGC), (-)-epigallocatechin 3-gallate (EGCG) and (-)-gallocatechin 3-gallate (GCG). While, the black tea polyphenols contained theaflavins such as theaflavin (TF-1), theaflavin 3-gallate (TF-2a), theaflavin 3'-gallate (TF-2b) and theaflavin 3,3'-digallate(TF-3) [5].

In order to understand the possible benefit of tea polyphenols in cancer prevention, MCF-7 human breast cancers cells were extracted and cultured with tea extracts from oolong, black, and green tea. in addition, the understanding of FAS upregulation in obesity and cancer has evaluated the contribution of several hormones and signal pathways involved in the upregulation of FAS gene expression such as sec hormones, epidermal growth factors (EGF), insulin, and phosphatidylinositol 3-kinase (PI3K)/Akt signal pathway [5].

The EGCG polyphenol from green tea and TF-3 from black tea have directly inhibited the FAS protein in the human body; in addition, to the suppression of EGF and insulin-induced FAS protein. Due to the contribution of PI3K/Akt signal pathway in the upregulation of FAS, an inhibitor of the signal pathway (Ly294002) was also evaluated, and showed nearly complete inhibition of FAS expression [5].

\subsection{Tomato Lycopenes}

The incidence of cancer is highly influenced by diet. It has been heavily emphasized that diets full of fruits and 
vegetables can decrease the risk of cancer. In addition, low levels of dietary carotenoids have been associated with certain types of cancers [6]. Carotenoids are a natural coloration to fruits and vegetables which give them the yellow, orange, or red pigmentation in the plant tissue [6]. They belong to the tetraterpene family and with more than 600 known variants. Of these variants, lycopene has been identified as a contributory anticancer property, which gives the red pigmentation in the ripe tomato fruits (Lycopersicon esculentum) [6].

Lycopene microarray analysis with breast cancer cells (MCF-7, MDA-MB-231, and MCF-10a) revealed several genetic pathways implicating apoptosis, cell cycle, and mitogen-activated protein kinase (MAPK) signaling pathways [6]. The apoptosis genes regulated through lycopene revealed involvement in the p53, caspase 8 , and tumor necrosis factor ligand. In addition, several genes were upregulated in DNA repair, including mutS homolog 6 (MSH6), mutL homolog 1 (MLH1), nonpolyposis type 2 (MLH1), RAD50 (MCF-10a), v-fos FBJ murine osteosarcoma viral oncogene homolog (FOS), mutS homolog 2 (MSH2), and damage-specific binding protein (DDB2) (MCF-7) [6,7].

In addition, microarray analysis also revealed that lycopene induced MAPK signaling transduction by regulating FOS, mitogen activated signal protein kinase 1 (MAP2K1), Janus kinase 1 (JAK1), v-jun sarcoma virus 17 oncogene homolog (JUN), signal transducer and activator of transcription 3 (STAT3) in MCF-7; BRCA1associated protein 1 (BAP1), Ras association (RaIGDS/ AF-6) domain family 7 (C11ORF13), mitogen activated protein kinase 14 (MAPK14) in MDA-MB-231; and tumor protein p53 (TP53) and protein kinase, DNA-activated, catalytic polypeptide (PRKDC) in MCF-10a [6,7].

Insulin-like growth factor 1 (IGF1) plays an important role in inhibiting endometrial, mammary, and lung cancer cell proliferation; however, with the addition of lycopene, IGF1 was downregulated in gene expression [6]. In addition, lycopene has shown to have upregulation of the gene GADD45 $\alpha$, which is involved in DNA damage repair, cell cycle checkpoint, and regulation of apoptosis pathways. In addition, BRCA1 has also been shown to be upregulated by lycopene, and induced beneficial apoptosis by activating Bcl-2 and GADD45 $\alpha$, and also have been shown to have additional benefits in damage repair and signaling [6].

Microarray analysis has also shown addition activation of damage repair and signaling pathways such as DDB2, MSH2, MSH3, BLM, MRE11A, SMC1L1, RAD50, MLH1 and MSH6, which are also mediated by BRCA1 and lycopene activation [6].

\subsection{Vitamin B9 (Folic Acid)}

Folate was extrapolated from spinach in 1941 by Mit- chell et al., and was shown that it plays an important role in DNA synthesis and methylation [8]. In addition, it acts as a single carbon donor, which can help in the regulation of cancer cells, through several mechanisms including the conversion of homocysteine to methionine and purine and pyrimidine synthesis $[8,9]$. It has shown that one of the folate-binding proteins, the human folate receptor (hFR), can become over expressed in some cancers such as leukemia, which made it an attractive candidate for anticancer therapy [8].

In the absence of folate in the diet, insufficient conversion of deoxyuridine monophosphate (dUMP) to deoxythymidine monophosphate (dTMP) can lead to misincorporation of uracil into the DNA strands, which results in increased double-strand breaks during uracil excision repair [8]. In addition, activated metabolites of fluoropyrimidines can also add to the aggregation of DNA damage and interfere with RNA synthesis. Rapidly multiplying cells are most susceptible to folate deficiency in the diet, and studies have revealed that gastrointestinal and hematopoietic cells are the most effected leading to liquid and solid cancers such as leukemia and stomach cancers [8].

Folate analogs have been introduced several years ago, with the most commonly known drug, methotrexate (MTX). MTX has been shown to be a very successful antitumor chemotherapy agent with immunosuppressive properties to treat leukemia, lymphoma, and other autoimmune diseases such as rheumatoid arthritis. It works primarily as an inhibitor for the dihydrofolate reductase (DHFR) which results in an accumulation of dihydrofolate (DHF) $[8,9]$.

Recent studies have shown genetic variations of methotrexate in its metabolism through several genetic pathways including 5,10-methylenetetrahydrofolate reductase (MTHFR), thymidine synthesis (TS), dihydrofolate reductase (DHFR), methionine synthase (MS), methionine synthase reductase (MTRR), cystathionine $\beta$-synthase (CBS), serine hydroxymethyltransferase (SHMT), and reduced folate carrier (RFC) $[8,9]$.

In-vitro studies have shown that MTHFR heterozygote carriers CT carried intermediate elevated plasma homocysteine levels as compared to the TT and CC homozygote alleles. This polymorphism has showed to be associated with different types of cancers including acute lymphocytic leukemia, colon cancer, neural tube defects, and possible cardiovascular disease. These types of diseases have been shown more likely in patients with low folate intake [8-10]. In addition, TS has been shown to be associated with double or triple repeats in the African American population, which resulted in an increased gene expression and increased levels of tumor tissue [8-10].

DHFR is the primary target of MTX, and in-vitro 
studies have shown a population-specific polymorphism first detected in the Japanese population. The results of these polymorphisms revealed an increased expression of DHFR with the homozygous TT genotype of the C829T gene, when compared to the heterozygote gene, CT, and the other homozygote allele CC $[9,10]$. In addition, individuals with MS deficiency have shown more likelihood to experience side effects such as hyperhomocysteinemia, homocystinuria, and megaloblastic anemia. Homocysteine levels have been linked to D919G, and in-vitro studies have revealed that individuals with the DD genotype experienced a higher homocysteine level, while the GG genotype experienced the lowest homocysteine level $[9,10]$.

MTRR deficiency has lead individuals to suffer a variety of disorders including deficiency in folate/cobalamin metabolism. In 1999, it was revealed that a substitution of methionine for isoleucine at codon 22 of gene A66G was the reason for the deficiency of the enzyme. In addition, AA homozygous individuals revealed increased homocysteine levels over the AG heterozygote, and GG homozygote allele $[9,10]$.

CBS deficiency results in homocystinuria; however, even though in-vitro studies revealed that polymorphisms from the substitution of the $\mathrm{C}$ to $\mathrm{T}$ allele on the nucleotide 699 and 1080, these polymorphisms acted as silent mutations $[9,10]$. Whereas, SHMT polymorphism in the C1420T allele of cSHMT gene revealed that red blood cells and folate levels were elevated in a small degree in individuals with the T allele (TT and TC) as compared to the CC homozygous alleles. Lastly, RFC polymorphism shows potential for pharmacogenomic relevance since it plays a major role in the entry of MTX into the cell; however, studies have not yet shown its significance $[9,10]$.

\subsection{Tobacco}

Tobacco is growth from the plant, Nicotiana tabacum [11], and has been widely used in cultivating cigarette products for tobacco smoking. It is estimated that $29 \%$ of the world's population over the age of 15 smokes, with harmful effects on the human cells affecting short-term memory, which in return affects attention [12]. In addition to memory, tobacco has been associated with nearly $30 \%$ of tumors, and involves several sites including lungs, head, neck, esophagus, stomach, liver, pancreas, bladder, kidney, and myeloid leukemia [13].

Lung cancer is considered one of the most fatal cancers, and the first tumor associated with it involved cigarette smoking.

The tumor size is influenced by many factors such as the number of cigarettes, age, pattern, type of tobacco, and several other factors [13]. While nicotine itself is not a carcinogenic, it leads to addiction and possible facilitation to other carcinogenic mechanisms such as angiogenesis. Tobacco carcinogens include polycyclic aromatic hydrocarbons (PAHs), such as benzo(a)pyrene, benz(a)anthracene and chrysene, N-nitrosamines, such as tobacco-specific nitrosamines (TSNA), 4-(methylnitrosamino)-1-(3-pyridyl)-butanone (NNK), and N-nitrosonornicotine (NNN), and lastly, aromatic amines [1113].

The metabolism of tobacco is dependent on the cytochrome P450 (CYP) enzymes. TSNA, nicotine, and other tobacco carcinogens are mainly activated by the CYP2A enzyme. The human CYP2A enzyme contains three types, which include the CYP2A6, CYP2A7, and CYP 2A13 [12,13].

CYP2A6 is the main active enzyme and mainly expressed in the liver, with other expression in other organs such as the nasal epithelium, trachea, lung and esophagus. The lung and esophagus have the highest expression of CYP2A6, with 50-fold and 41-fold, respectively. While the CYP2A13 is mainly expressed in the nasal epithetlium compared with the other organs. However, the expression of CYP2A6 remains the most active enzyme in the human body compared to the other enzyme derivatives [11-13].

Nicotine is viewed as a pro-drug, and requires the metabolism of 5'-hydroxylation to convert it to its active metabolite cotinine. Cotinine can be further metabolized in the body by trans-3'-hydroxycotinine, which is the main compound found in urine $[12,13]$. While, In vitro-studies have correlated CYP2A13 expression in nicotine metabolism to cotinine, the expression of CYP2A6 in the main conversion of nicotine to cotinine in the human liver, which also participates in further converting cotinine to trans-3'-hydroxycotinine for urinary excretion. CYP2A6 can be heavily influenced by genetic composition, diet, and environment, which can lead an individual to become a CYP2A6 inhibitor or inducer [12].

In addition to nicotine conversion, $\mathrm{NNK}$ and $\mathrm{NNN}$ carcinogens have also assisted in the expedition to cancerous formation while also depending on the CYP2A enzyme metabolism pathway. It is understood that NNK can be metabolized to two metabolites, $\alpha$-methyl or $\alpha$ methylene hydroxylation, depending on the tissue it encounters. The expression of the different CYP2A enzymes has also influenced the conversion of the carcinogens [13]. The $\alpha$-methyl pathway is metabolized by the CYP2A6 enzyme pathway, while the $\alpha$-methylene hydroxylation is metabolized through the CYP2B6 and CYP2A13 enzyme pathway, leading researchers to acknowledge that $\alpha$-methyl is the main metabolism pathway for nicotine due to its direct association with CYP2A6 [13]. 
NNN can also become metabolized at the 2'- and 5 '-hydroxylation pathway, noting that the 5'-hydroxylation is considered the main stream pathway in humans, which is carried out by the cartelization of the CYP2A6 and CYP2A13 [12].

Genetic factors play an important part in the likelihood of smoking tobacco. Genetic polymorphisms in the enzyme activity of CYP2A6 can influence individuals to smoke more or less frequently depending on the copies found in their genetic profile. Individuals who carry extra copies of the CYP2A6 gene are considered fast metabolized, and are considered to be susceptible to heavier smoking due to increased nicotine clearance from the body.

In addition, individuals who carry inactive or reduced CYP2A6 alleles can benefit, since their metabolism is heavily slowed down. The inactive alleles are encoded as CYP2A6* 2 , CYP2A6 ${ }^{*} 4$ and CYP2A6 ${ }^{*} 20$, while the reduced enzyme activation are encoded as CYP2A6 ${ }^{*} 7$, CYP2A6* 10 and CYP2A6*17 [12]. These genetic factors have been shown to be influenced by ethnicity, as in-vitro studies have showed that even though African-American individuals smoked as much as European-Americans, their serum concentration for nicotine was nearly $50 \%$ and $30 \%$ of nicotine and cotinine per cigarette intake, respectively, which showed that African-Americans have a genetic predisposition for lower nicotine clearance from the body $[12,13]$. In addition, Nakajima et al., and showed that Japanese nicotine and concentrations in the body were higher than African Americans and European Americans, while J. T. Kwon, et al. showed the same predisposition with Koreans. These studies suggest that ethnic background carry a predisposition to the likelihood of heavy smoking according to their genetic allele [12].

These studies have led to more investigational drug discovery to make new drugs that target the inhibition of the CYP2A6 metabolic pathway. It has been observed through the use of 8-methoxypsoralen, a CYP2A6 inhibitor, and it showed that individuals who carried this gene had a higher nicotine plasma concentration in the body, which resulted in nearly $24 \%$ reduction in the number of cigarette smoked per day, an $84 \%$ increase in the intervals between each cigarette to the next, and a $25 \%$ reduction in the total amount of puffs per day [1113].

Methoxsalen (8-methoxypsoralen), a CYP2A6 inhibitor, was introduced into the market suggesting a possible beneficial affect for individuals consuming this product. In addition, the drug proposes addition benefits since CYP2A6 does not have a major role in the body, eliminating any drug interactions or unwanted side effects [13].

\subsection{Kampo and the Use of Shikonin}

Kampo has been used in Chinese culture as a natural medical plant by monks. Its use has also been documented in Japanese emperor's courts. The chemical components of the medicinal plant have been used mainly as a compounded agent with other medicinal plant, giving it the wide array of use in medicine. The use of kampo increased further as a supportive agent for chemotherapy individuals, since it promotes physical reconditioning and reduces the side effects of chemotherapy [14].

A molecular analysis was conducted using different Chinese medicinal plants, and it was determined that Shikonin possessed anticancer properties against mRNA expression. It is commonly known as zicao, and derived as the dried root of plant species Lithospermum erythrorhizon $[15,16]$. The microanalysis showed that Shikonin several cancer pathways, such as mRNA suppression, DNA topoisomerase I and II, and bioreductive alkylation, increased concentrations of p27 and p53, and decreased bcl-2 and Bcl-XL leading to apoptosis which can help target cancer genes [14-16]. In addition, it showed that the medical plant had effects at the G0/G1 phase of the cancer cell cycle, which induces apoptosis of cancer genes in the early stage of the cycle. It has also showed that the use of this herb did not show any side effects for lung cancer patients treated with zicao [16].

In order to further understand the effects of zicao in chemotherapy, an in-vitro analysis was conducted by Yuan Yao, et al. examining its effects on estrogen in breast cancer patients. The results of the study showed the benefit of zicao in several anticancer inhibitory pathways including suppression of ER $\alpha$-dependent gene transcription, ER $\alpha$-positive breast cancer cells further verifying its beneficial effects as its use in anti-cancer therapy [16].

\section{Cardiovascular Diseases}

\subsection{Tea Polyphenols}

Chemical extracts from green and black tea were extracted and fed in a prospective clinical trial to examine the effects of tea polyphenols in anti-obesity effects. $2.5 \%$ pulverized green tea leaves were for to rats for 63 weeks, and the results showed that in addition to neutral effects of tea leaves to the liver and kidney of the rats, body weight reduction and hypolipidemia was observed. The LDL-cholesterol, total cholesterol, and triglycerides were reduced in the treated group compared to the control group [5].

In addition, in-vitro studies have shown the clinical benefit of the green tea polyphenol EGCG, which showed to have a similar mechanism to the effects of 
leptin, acting as a FAS-inhibitor in the body [5].

\subsection{Vitamin $E$}

Cardiovascular diseases have attributed to several complications including diabetes mellitus, hypertension, increased risk of stroke and myocardial infarction, and even cancer. Diabetes mellitus with cardiovascular diseases increases the burden of patient health through increased free radical oxygen species in the body. Although several clinical trials did not show that additional benefit of Vitamin E helps patients, it left researchers to investigate the possible polymorphism in the genetic profile of patients [17].

Cardiovascular disease also leads patients to experience oxidative stress, which promotes further production of highly reactive oxygen species (ROS). They contain unpaired electrons, which can further aggravate the body through its involvement in intracellular signaling. It can also result in several chain reactions that cause significant damage to the human biological molecules such as DNA, proteins, carbohydrates, and lipids. ROS has been directly related to the development of atherosclerosis, which happens due to attributing the electron particle of ROS to the low-density lipoprotein (LDL) causing it to become an oxidized LDL (ox-LDL), which is taken up by CD36 scavenger receptors in the macrophages leading its conversion to foam cells [18].

It has been documented that the level of ROS is increased in diabetes mellitus patients, and hyperglycemia adds to the additional ROS generation. Antioxidant compounds have been suggested as potential cure for certain ROS species, while Vitamin E concentrations are decreased in diabetes mellitus patients [18]. Vitamin E comes in a synthetic and natural form. The synthetic form consists of both the D- and L-stereoisomer, while the naturally occurring Vitamin $\mathrm{E}$ only contains the D-stereoisomer which still contains a high antioxidant property [18]. The most abundant form of Vitamin E is the $\alpha$-tocopherol, and it is most prevalent in naturally occurring forms of Vitamin E, and acts as an antioxidant agent against the cells. It was shown that it can react with peroxyl radicals to form lipid hydroperoxide and the tocopheroxyl radical, which is a relatively stable and unreactive with the body's constituents [18].

One polymorphism that could affect the patient response was the haptoglobin (Hp) genetic locus produced in the liver and binds to free hemoglobin $(\mathrm{Hb})$ released from the blood. It prevents the $\mathrm{Hb}$ from participating in oxidative injury in the human body in the hepatic site; however, in extrahepatic sites, it can also serve as a stress factor for the body, by preventing the release of heme iron in the body due to stringent binding, which leads to the formation of the Hb-Hp complex. The complex can- not be cleared by the liver, and can only rely on the macrophages, which in return, transforms into foam cells [18].

The Hp gene contains several louses including the Hp1 (Hp1-1 allele), which is more prevalent in Western populations, and the Hp2 gene (Hp2-2 and Hp2-1 alleles), the later is present only in humans and provides less beneficial effects of antioxidant protection against $\mathrm{Hb}$ when compared to Hp1 [17]. Hp polymorphism has been documented as a major determinant for determining the risk of developing cardiovascular diseases in diabetes mellitus patients. However, the increased risk of cardiovascular disease was most prevalent in patients who possessed the Hp-2 gene, with the highest risk for individuals who carried the Hp2-2 allele [17]. Individuals with the Hp-2 gene experienced more risk for developing diabetic retinopathy, diabetic nephropathy, and atherosclerosis compared to those who carried the Hp-1 gene [18].

Vitamin E supplementation (400 IU per day) was given to diabetes mellitus patients with $\mathrm{Hp} 1$ and $\mathrm{Hp} 2$ polymorphisms and investigated for risk of myocardial infarction and stroke. The Hp2-2 group who received Vitamin E significantly benefited from the added supplement, and consequently a significant reduction in the number of cardiovascular events by over $40 \%$. In addition, Vitamin E supplementation has shown to reduce atherosclerotic lesion size and progression, platelet adhesion, and protein kinase $\mathrm{C}$ activation [18]. In addition, the use of Vitamin C showed to have a harmful effect on the genotype, increasing the oxidative activity of the lipids and blocked the activity of Vitamin E to correct HDL dysfunction [17]

\subsection{Curcumin}

Curcumin (diferuloylmethane) is the yellowish component of curry, which is a popular culinary meal in the Asian sub- constituent, especially in the Indian culture. It a polyphenolic compound extracted from the rhizome of the Curcuma longa L. (family Zingiberaceae) [19]. It has been studied excessively and showed potential for its use as an anti-inflammatory, anti-oxidative, anti-vial, and anti-hypercholesterolemic agent. In several in-vitro studies, it has been shown to inhibit hepatic stellate cell (HSC) activation, with induction of the PPAR $\gamma$ and sterol regulatory gene element binding proteins (SREBPs), leading to a reduction in the cholesterol [20].

Ox-LDL is the major lipid component leading to hyperlipidemia, which predisposes individuals to suffer from additional cardiovascular complications including hypertension, stroke, and myocardial infarction. Lipids are mediated in the body through several mechanisms and scavenger receptors, including the LOX-1, SR-AI/II, CD36, and SR-BI [20]. The expression of each scavenger 
receptor relies on specific tissues in the body, such as CD36's high expression in macrophages, which plays a major role in LDL cholesterol found in human macrophages. LOX-1 is the main receptor signal pathway for ox-LDL cholesterol, which is found primarily in the human epithelial cells. It also plays a major role in converting ox-LDL into HSC [20].

LOX-1 is a scavenger receptor, which facilitates the accumulation of ox-LDL, and stimulates the human cells to transform macrophages into foam cells increasing the cholesterol level in the body. In order to further understand the effects of curcumin, HSCs were cultured with human ox-LDL. Curcumin was shown to suppress the LOX-1 expression [20].

The main beneficial effect of curcumin was due to its direct effect on the expression of PPAR $\gamma$. The activation of this signal pathway added to an additional inhibition of the LOX-1 gene expression, through the increased expression of the canonical Wnt signaling, which mediated the effects of LOX-1 suppression. In addition, through dose-dependent curcumin, it showed that it can directly affect the Wnt signaling, and decreased the DNA binding of Wnt signaling pathways [20].

\section{Osteoporosis}

\subsection{Soy}

Estrogen plays a critical role in bone homeostasis. The receptors of estrogen are expressed in the osteoblasts and osteoclasts. In osteoclasts, they play a role in inhibiting interleukin (IL) 1, IL6, IL7, tumor necrosis factor- $\alpha$, and the receptor activator of nuclear factor (NF) $-\kappa \mathrm{B}$ ligand [21]. In addition, osteoblasts help promote a longer lifespan by inhibiting their apoptosis. The beneficial effects of estrogen in bone formation can also be proven by the increased bone mineral density loss in female patients after the menopause, since ovarian production of estrogen is significantly decreased [21].

Aromatase is involved in the conversion of C-19 androgens into estrogens. It is the last process of estrogen production, which converts the androstenedione and testosterone into the C-18 estrogens. It is expressed in the gonads and plays a critical role in the ovarian synthesis of estradiol [21]. The aromatase enzyme is also expressed in several other tissues including fat, placenta, breast, skin and bone. Estrogen involvement in bone formation and stability has been associated with its interaction with the osteoblastic lineage and chondrocytes. Estrogen contains many precursors in the body, with estradiol being the most active compound among the natural estrogens [21]. The enzyme is found in the endoplasmmic reticulum and expressed through the cytochrome P450, primarily through the CYP19A1. Several polymorphisms have been expressed; the most widely known polymorphism is the TTTA repeat sequence in intron 4, which is also linked the TTC insertion/deletion polymorphism. The TTTA short alleles have been found to have lower BMD in postmenopausal women $[21,22]$.

Soy has been viewed as a natural estrogen replacement for postmenopausal women. It contains several phytoestrogens, primarily genistein, which shows slowed rate of bone loss formation through several protein synthesis, such as protein synthesis in osteoblast-like cells [23]. Dietary soy has been investigated and showed similar effects of reduced bone density through suppressed bone turnover. In addition, phytoestrogens in soy have been found to be endoplasmic reticulum (ER)- $\beta$ selective, and bind to it with a higher selectively than the strongest form of estrogen, estradiol, indicating that soy may exert its effects in a separate and distinct form than traditional estrogen [23].

\subsection{Vitamin D}

The major source of Vitamin D is the human skin. It is produced through the action of ultraviolet (UV) light on steroid receptors. It is also found in natural substances such as dietary food, and can help patients when there is insufficient UV exposure. It also correlates with the decreased dietary calcium and sunlight exposure of elderly, and highly suggestive of different levels of Vitamin D depending on the age, skin pigmentation, season and sun exposure a certain population might experience [24]. It can be derived from plant substances, with a chemical composition of Vitamin D2, ergocalciferol, and animal sources, with a chemical composition of Vitamin D3, cholecalciferol. It is absorbed in the small intestine in the presence of bile acid [24].

Despite its name, it is not a true Vitamin, but a pro-steroid hormone that is metabolized into 23-hydroxychlecalciferol (25OHD), which acts as the major storage form. The active metabolite of vitamin D, calcitriol (1.25 $(\mathrm{OH}) 2 \mathrm{D})$, is metabolized in the kidney, and it's the main hormone responsible for the biological effects of Vitamin D. when calcitriol binds to the Vitamin D receptor (VDR) to regulate gene transcription of several target tissues such as bone, intestine, kidney, and parathyroid glands. Calcitriol exerts its function differently depending on the target tissue; in the intestine, calcitriol induces the expression of calcium to help circulate the dietary calcium into the circulation. While on the bone, it is thought that osteoblasts secrete cytokines that stimulate osteoclast differentiation and cone resorption in response to calcitriol formation [24].

The VDR gene has been proposed as a possible explanation for increased osteoporosis risk. The gene is located on the long arm of chromosome 12 and composed of nine exons, with eight transcribing exons that 
are translated into function CDR proteins. Polymorphisms in the restriction enzymes BsmI and ApaI have been documented, with increased osteocalcin levels in individuals with the "BA" allele [24,25]. The "bb" genotype is characterized by the absence of the restriction site for $B s m I$, and it was correlated with the highest BMD value with the lowest fracture risk, while the "BB" genotype was typical of women who are just below the threshold of osteoporotic fracture risk. In addition, haplotype alleles of the VDR gene associated with fracture risk has been also associated with decreased body height, decreased bone size, and lower bending strength at the femoral neck [24].

It has been shown that BsmI VDR polymorphism and calcium intake encoding the bb homozygote allele showed a higher BMD, yet no effect during calcium and Vitamin D supplementation in comparison to the $\mathrm{Bb}$ genotype, crossing from net loss to net gain when consuming approximately $1 \mathrm{~g} /$ day. In addition, a study was conducted in postmenopausal Normal American women, and it supported that women with the BB genotype showed decreased calcium absorption when compared to the $\mathrm{bb}$ and $\mathrm{Bb}$ genotypes. Given that the $\mathrm{B}$ genotype is more prevalent in the European ancestry, it could partly explain the responsive differences between studies conducted in Asians and Europeans and their response to calcium and Vitamin D supplementation [25].

\section{Depression}

\subsection{Soy}

Stress and sex hormones play a vital role in elevating mood. The serotonin (5-HT) is one of the main neurotransmitters for mood stabilization, and several environmental factors as well as genetics can affect its level in the human body. 5-HT is controlled by several neurotransmitters and regulatory proteins, mainly tryptophan hydroxylase (TPH), the committal enzyme in 5-HT synthesis, and the 5-HT reuptake transporter (SERT), which has been a target for several antidepressant medications such as the selective serotonin reuptake inhibitors (SSRIs) [26].

Depression has been more common in females, suggesting that the elevated estrogen level from the men strual phase could be attributing to its elevation. Estrogen has been an important factor in reducing several diseases and regulating the menstrual cycle of women; however, artificial supplementation leaves the patient with unwanted side effects. It was suggested that natural supplementation of estrogen such as soy phytoestrogen (SPE) could benefit female patients since it does not have either agonist or antagonist activity [26].

SERT has been the main regulatory serotonin protein transmitter in the body, and any dysfunction can lead to depression or anxiety disorders, as it was documented that the SERT levels of patient with major depression was reduced in the midbrain; however, one month of estrogen replacement helped the SSRI, citalopram, bind more effectively and produce better results for patients [26].

In-vitro research have suggested the beneficial effect of adding soy isoflavone supplements since it antagonized the estrogen receptor- $\alpha$ and $\beta$-dependent gene expression in the hypothalamus. They also increased the choline acetyltransferase mRNA levels and the mRNA neurotrophic factor in the frontal cortex [26]. It was also shown that SPE can also increase TPH protein in the serotonin body similar to that of conjugated equine estrogens, which is common estrogen product used for hormone replacement therapy. In addition, males could benefit from the added dietary soy products since phytoestrogens was shown to decrease medical basal hypothalamus and amygdaloid calcium-binding proteins without interfering with the androgen metabolizing enzymes [26].

\subsection{Curcumin}

In addition to the beneficial cardiovascular effects of curcumin, it was shown that the dietary supplementation also acts as an inhibitor of the transcription factor AP-1, a gene expression regulatory factor expressed in lithium treatment [27]. In addition, it was shown that curcumin helped elevate the brain levels of serotonin $[19,28]$. It was also shown that at doses of $10-80 \mathrm{mg} / \mathrm{kg}$, demonstrated a synergistically enhanced effect of tranylcypromine (monoamine oxidase inhibitor), fluoxetine (selective serotonin reuptake inhibitor), bupropion (dopamine reuptake inhibitor), and venlafaxine (dual reuptake inhibitor of serotonin and norepinephrine) [19]. However, in-vitro studies have not shown a positive correlation with curcumin addition to desipramine and imipramine (tricyclic antidepressant) [19].

Dopamine levels are also considered a critical component of antidepressant effects and in-vitro studies have shown that curcumin also played a role in increasing dopamine level through MAO-B enzyme activity. It suggested that curcumin potentiates the same effects as MAO inhibitors [28]. It was also observed that curcumin can inhibit both the MAO-A and MAO-B receptors; although higher doses were required to inhibit the MAO-B receptors. The MAO-A receptors were shown to be inhibited by low doses, with higher doses inhibiting the MAO-B receptors with no effect on the norepinephrine in the brain [28].

\section{Atrial Fibrillation}

\section{Digoxin}

Digitalis have been used in the early 20th century as a 
useful medicinal agent for patients with heart failure, it was later synthesized into digoxin to treat patients with atrial fibrillation [29]. MDR1 gene encodes for the ATPdependent membrane efflux transport for p-glycoprotein, and it was shown that MDR1 polymorphisms could affect the pharmacokinetics of digoxin [30,31]. The P-gp acts as a pump to remove substrates from inside to the outside of the cell. It is expressed in several human tissues such as the liver, kidney, small and large intestine, brain, placenta, and adrenal glands [31].

In-vitro studies have been conducted, and it was shown that homozygous wide-type individuals with the $\mathrm{CC}$ allele are considered to be high expressers for the P-gp, which requires low digoxin levels, while those who had the homozygous TT allele with low expressers of the $\mathrm{P}$-gp, and required higher doses of digoxin for controlled benefits [30]. It was shown in urinary recovery of patients who were treated with digoxin that $\mathrm{TT}>\mathrm{CT}>\mathrm{CC}$ supported previous studies that suggested that individuals with the TT allele required higher digoxin doses due to low levels of the intestinal P-gp [31,32]. However, it should not be the only determinant of the expression since environmental factors such as diet may explain the differences between intestinal expressions of the P-gp gene [31].

In addition, it was observed that the C3435T and G2677T alleles were found in high frequencies with the MDR1 polymorphism; however, several in-vitro studies that found no correlation between C3435T on the MDR1 expression based on several different organs such as the placenta and duodenal tissues with different ethnic background including Japanese and Caucasians [31]. Unlike C3435T allele, the G2677T showed a reduced accumulation of the digoxin levels compared to the wild type, suggested that there might be an enhanced efflux effect with G2677T [31].

\section{Future Perspectives}

It is well recognized that different patients express differently to medications according to several factors such as genetics, environment, and food intake. Genetic variability between individuals and their response to medications and food could dramatically affect their response however, this area of research is relatively new and requires several years to decode the human body genomes and understand its influence with the environment [31].

There have been several issues with many researchers and the public view on the use of genetics to treat patients, judging the freedom, justice, health safety, and privacy as a few of the main concerns people might face with the availability of pharmacogenomics [33]. However, several researchers support the aim behind pharmacogenomics research emphasizing that the genetic data of individuals will not be used to discriminate against individuals since they have full control over knowing their genetic data [33].

The area of pharmacogenomics is still in its infancy; however, it shows great potential for to understand the significance behind the genetics of transporters, allele polymorphisms, and food interactions to enhance the wellbeing and knowledge of patients.

\section{REFERENCES}

[1] I. S. Vizirianakis, "Improving Pharmacotherapy Outcomes by Pharmacogenomics: From Expectation to Reality?" Pharmacogenomics, Vol. 6, No. 7, 2005, pp. 701711. doi:10.2217/14622416.6.7.701

[2] U. Mahlknecht and S. Voelter-Mahlknecht, "Pharmacogenomics: Questions and Concerns," Current Medical Research and Opinion, Vol. 21, No. 7, 2005, pp. 1041-1047. doi:10.1185/030079905X50633

[3] H.-F. Ji, X.-J. Li and H.-Y. Zhang, "Natural Products and Drug Discovery," EMBO Reports, Vol. 10, No. 3, 2009, pp. 194-200. doi:10.1038/embor.2009.12

[4] P. Vuorelaa, et al., "Natural Products in the Process of Finding New Drug Candidates," Current Medicinal Chemistry, Vol. 11, No. 11, 2004, pp. 1375-1389.

[5] C.-W. Yeh, et al., "Suppression of Fatty Acid Synthase in MCF-7 Breast Cancer Cells by Tea and Tea Polyphenols: A Possible Mechanism for Their Hypolipidemic Effects," The Pharmacogenomics Journal, Vol. 3, 2003, pp. 267276.

[6] N. Chalabi, et al., "Gene Signature of Breast Cancer Cell Lines Treated with Lycopene," Pharmacogenomics, Vol. 7, No. 5, 2006, pp. 663-672. doi: $10.2217 / 14622416.7 .5 .663$

[7] P. S. Rai, et al., "Genetic Variation in Genes Involved in Folate and Drug Metabolism in a South Indian Population," Indian Journal of Human Genetics, Vol. 17, No. 1, 2011, pp. 48-53.

[8] C. M. Ulrich, et al., "Pharmacogenetics and Folate Metabolism-A Promising Direction," Pharmacogenomics, Vol. 3, No. 3, 2002, pp. 299-313. doi: $10.1517 / 14622416.3 .3 .299$

[9] M. Krajinovic, et al., "Role of Polymorphisms in MTHFR and MTHFD1 Genes in the Outcome of Childhood Acute Lymphoblastic Leukemia," The Pharmacogenomics Journal, Vol. 4, 2004, pp. 66-72. doi:10.1038/sj.tpj.6500224

[10] D. E. Riechers and P. T. Michael, "Structure and Expression of the Gene Family Encoding Putrescine N-Methyltransferase in Nicotiana Tabacum: New Clues to the Evolutionary Origin of Cultivated Tobacco," Plant Molecular Biology, Vol. 41, No. 3, 1999, pp. 387-401. doi:10.1023/A:1006342018991

[11] A. Rigbi, et al., "Why Do Young Women Smoke? VI. A Controlledstudy of Nicotine Effects on Attention: Pharmacogenetic Interactions," The Pharmacogenomics Journal, Vol. 11, No. 1, 2011, pp. 45-52.

doi:10.1038/tpj.2010.15 
[12] A. Rossini, et al., "CYP2A6 Polymorphisms and Risk for Tobacco-Related Cancers," Pharmacogenomics, Vol. 9, No. 11, 2008, pp. 1737-1752. doi:10.2217/14622416.9.11.1737

[13] J. K. Yano, et al., "Structures of Human Microsomal Cytochrome P450 2A6 Complexed with Coumarin and Methoxsalen," Nature Structural and Molecular Biology, Vol. 12, No. 9, 2005, pp. 822-823. doi:10.1038/nsmb971

[14] T. Errerth, et al., "Pharmacogenomics of a Traditional Japanese Herbal Medicine (Kampo) for Cancer Therapy," Cancer Genomics and Proteomics, Vol. 4, 2007, pp. 8192.

[15] X. Chen, et al., Shikonin, a Component of Chinese Herbal Medicine, Inhibits Chemokine Receptor Function and Suppresses Human Immunodeficiency Virus Type 1," Antimicrob Agents Chemother, Vol. 47, No. 9, 2003, pp. 2810-2816. doi:10.1128/AAC.47.9.2810-2816.2003

[16] Y. Yuan and E. Q. Zhou, "A Novel Antiestrogen Agent Shikonin Inhibits Estrogen-Dependent Gene Transcription in Human Breast Cancer Cells," Breast Cancer Research and Treatment, Vol. 121, No. 1, 2010, pp. 233240. doi:10.1007/s10549-009-0547-2

[17] S. Blum, et al., "Vitamin E Reduces Cardiovascular Disease in Individuals with Diabetes Mellitus and the Haptoglobin 2-2 Genotype," Pharmacogenomics, Vol. 11, No. 5, 2010, pp. 675-684. doi:10.2217/pgs.10.17

[18] A. P. Levy and S. Blum, "Pharmacogenomics in Prevention of Diabetic Cardiovascular Disease: Utilization of the Haptoglobin Genotype in Determining Benefit from Vitamin E," Expert Review of Cardiovascular Therapy, Vol. 5, No. 6, 2007, pp. 1105-1111. doi:10.1586/14779072.5.6.1105

[19] P. N. Mimche, et al., "The Plant-Based Immunomodulator Curcumin as a Potential Candidate for the Development of an Adjunctive Therapy for Cerebral Malaria," Malaria Journal, Vol. 10, No. 1, 2011, pp. 1-9. doi:10.1186/1475-2875-10-S1-S10

[20] Q. H. Kang and A. P. Chen. "Curcumin Eliminates Oxidized LDL Roles in Activating Hepatic Stellate Cells by Suppressing Gene Expression of Lectin-Like LDL Receptor-1," Laboratory Investgation, Vol. 89, No. 11, 2009, pp. 1275-1290. doi:10.1038/labinvest.2009.93

[21] J. A. Riancho, "Polymorphisms in the CYP19 Gene that Influence Bone Mineral Density," Pharmacogenomics, Vol. 8, No. 4, 2007, pp. 339-352. doi: $10.2217 / 14622416.8 .4 .339$

[22] S. C. Blum, et al., "Dietary Soy Protein Maintains Some Indices of Bone Mineral Density and Bone Formation in
Aged Ovaricetomized Rats," The Journal of Nutrition, Vol. 133, No. 5, 2003, pp. 1244-1249.

[23] L. Gennari, et al., "Update on the Pharmacogenetics of the Vitamin D Receptor and Osteoporosis," Pharmacogenomics, Vol. 10, No. 3, 2009, pp. 417-433. doi:10.2217/14622416.10.3.417

[24] F. Massart, "Human Races and Pharmacogenomics of Effective Bone Treatments," Gynecological Endocrinology, Vol. 20, No. 1, 2005, pp. 36-44. doi:10.1080/09513590400019437

[25] C. A. Shively, et al., "Soy and Social Stress Affect Serotonin Neurotransmission in Primates," The Pharmacogenomics Journal, Vol. 3, No. 2, 2003, pp. 114-121. doi:10.1038/sj.tpj.6500166

[26] T. Hiroi, et al., "Protracted Lithium Treatment Protects against the ER Stress Elicited by Thapsigargin in Rat PC12 Cells: Roles of Intracellular Calcium, GRP78 and Bcl-2," The Pharmacogenomics Journal, Vol. 5, 2005, pp. 102-111.

[27] S. K. Kulkarni and A. Dhir, "An Overview of Curcumin in Neurological Disorders," Indian Journal of Pharmaceutical Sciences, Vol. 72, No. 2, 2010, pp. 149-154. doi:10.4103/0250-474X.65012

[28] S. Kulkarni, et al., "Antidepressant Activity of Curcumin: Involvement of Serotonin and Dopamine System," Psychopharmacology, Vol. 201, No. 3, 2008, pp. 435-442. doi:10.1007/s00213-008-1300-y

[29] E. Erichorn, et al., "Digoxin - New Perspective on an Old Drug," The New England Journal of Medicine, Vol. 347, No. 18, 2002, pp. 1394-1395. doi:10.1056/NEJMp020118

[30] C. Verstuyft, et al., "Digoxin Pharmacokinetics and MD R1 Genetic Polymorphisms," European Journal of Clinical Pharmacology, Vol. 58, No. 12, 2003, pp. 809-812.

[31] G. D. Leschziner, et al., "ABCB1 Genotype and PGP Expression, Function and Therapeutic Drug Response: A Critical Review and Recommendations for Future Research," The Pharmacogenomics Journal, Vol. 7, No. 3, 2007, pp. 154-179. doi:10.1038/sj.tpj.6500413

[32] T. Sakaeda, et al., "Pharmacogenetics of MDR1 and Its Impact on the Pharmacokinetics and Pharmacodynamics of Drugs," Pharmacogenomics, Vol. 4, No. 4, 2003, pp. 397-410. doi:10.1517/phgs.4.4.397.22747

[33] N. W. Paul and A. D. Roses, "Pharmacogenetics and Pharmacogenomics: Recent Developments, Their Clinical Relevance and Some Ethical, Social, and Legal Implications," Journal of Molecular Medicine, Vol. 81, No. 3, 2003, pp. 135-140. 